\title{
Attention Configures Synchronization Within Local Neuronal Networks for Processing of the Behaviorally Relevant Stimulus
}

\section{OPEN ACCESS}

Edited by:

Fu-Ming Zhou,

University of Tennessee Health Science Center, United States

Reviewed by:

Ernst Niebur,

Johns Hopkins University,

United States

Ekaterina Levichkina,

The University of Melbourne, Australia

*Correspondence:

Eric Drebitz

drebitz@brain.uni-bremen.de

†Present Address:

Marcus Haag,

Institute of Neuroscience,

Newcastle University,

Newcastle-upon-Tyne,

United Kingdom

Iris Grothe,

Ernst Strüngmann Institute (ESI) for

Neuroscience in Cooperation with Max Planck Society, Frankfurt,

Germany

Received: 22 March 2018 Accepted: 09 August 2018

Published: 29 August 2018

Citation:

Drebitz E, Haag M, Grothe I,

Mandon S and Kreiter AK (2018) Attention Configures Synchronization

Within Local Neuronal Networks for

Processing of the Behaviorally

Relevant Stimulus.

Front. Neural Circuits 12:71.

doi: 10.3389/fncir.2018.00071

\author{
Eric Drebitz*, Marcus Haag ${ }^{\dagger}$, Iris Grothe ${ }^{\dagger}$, Sunita Mandon and Andreas K. Kreiter \\ Center for Cognitive Science, Brain Research Institute, University of Bremen, Bremen, Germany
}

The need for fast and dynamic processing of relevant information imposes high demands onto the flexibility and efficiency of the nervous system. A good example for such flexibility is the attention-dependent selection of relevant sensory information. Studies investigating attentional modulations of neuronal responses to simultaneously arriving input showed that neurons respond, as if only the attended stimulus would be present within their receptive fields (RF). However, attention also improves neuronal representation and behavioral performance, when only one stimulus is present. Thus, attention serves for selecting relevant input and changes the neuronal processing of signals representing selected stimuli, ultimately leading to a more efficient behavioral performance. Here, we tested the hypothesis that attention configures the strength of functional coupling between a local neuronal network's neurons specifically for effective processing of signals representing attended stimuli. This coupling is measured as the strength of $\gamma$-synchronization between these neurons. The hypothesis predicts that the pattern of synchronization in local networks should depend on which stimulus is attended. Furthermore, we expect this pattern to be similar for the attended stimulus presented alone or together with irrelevant stimuli in the RF. To test these predictions, we recorded spiking-activity and local field potentials (LFP) with closely spaced electrodes in area V4 of monkeys performing a demanding attention task. Our results show that the $\gamma$-band phase coherence ( $\gamma$-PhC) between spiking-activity and the LFP, as well as the spiking-activity of two groups of neurons, strongly depended on which of the two stimuli in the RF was attended. The $\gamma$-PhC was almost identical for the attended stimulus presented either alone or together with a distractor. The functional relevance of dynamic $\gamma$-band synchronization is further supported by the observation of strongly degraded $\gamma$-PhC before behavioral errors, while firing rates were barely affected. These qualitatively different results point toward a failure of attention-dependent top-down mechanisms to correctly synchronize the local neuronal network in V4, even though this network receives the correctly selected input. These findings support the idea of a flexible, demand-dependent dynamic configuration of local neuronal networks, for performing different functions, even on the same sensory input.

Keywords: visual cortex, macaque monkey, gamma-band, area V4, neuronal network configuration, spatial selective attention, functional coupling, dynamic assembly formation 


\section{INTRODUCTION}

Successful and goal directed behavior within our complex world requires a dynamic and adaptive processing of relevant information. Thus, local neuronal networks are required to perform different functions depending on the current requirements of information processing. A well-known example for such adaptation of network functions is the attentiondependent selection of different subsets of afferent input for effective processing of relevant visual information. Several studies investigating responses of neurons to simultaneous and convergent input showed that neurons responded almost as if only the attended stimulus would be present within their receptive field (RF) (Moran and Desimone, 1985; Treue and Maunsell, 1996; Luck et al., 1997; Reynolds et al., 1999; Lee and Maunsell, 2010; Grothe et al., 2012). Such changes of network function quickly follow changing behavioral demands although anatomical connections cannot change on such short timescales (Gilbert and Sigman, 2007; Carandini and Heeger, 2012; Park and Friston, 2013). However, recent theoretical work indicates that modulating $\gamma$-band synchronization can change the strength of the functional coupling between neuronal populations. Selective synchronization of a local network with only those upstream inputs representing the attended stimulus could therefore constitute a mechanism underlying this attention-dependent bias of neuronal responses (Börgers and Kopell, 2008; Battaglia et al., 2012; Wildie, 2012; Hahn et al., 2014; Harnack et al., 2015; Palmigiano et al., 2017). We and others showed indeed that the strength of $\gamma$-band phase coherence $(\gamma$-PhC) between $\mathrm{V} 1$ and V4 populations with overlapping receptive fields (RF) was much stronger when the $\mathrm{V} 1$ population represented the attended instead of the non-attended stimulus (Bosman et al., 2012; Grothe et al., 2012, 2018). These theoretical and experimental results indicate that attention-dependent selective processing of relevant afferent input depends on the strength of functional coupling between neurons along the visual pathway.

Yet, attention also modulates perception and neuronal responses when there is no competition between inputs. With only one stimulus inside the RF, attention has been shown to increase firing rates (Treue and Maunsell, 1996; McAdams and Maunsell, 1999; Reynolds et al., 2000; Wegener et al., 2004), to reduce the response variability of individual neurons (Mitchell et al., 2007; Galashan et al., 2013; Schledde et al., 2017) and to reduce the shared response variability of neuronal populations (Cohen and Maunsell, 2009; Mitchell et al., 2009; Schledde et al., 2017). Thus, besides the selective routing of relevant information, attention also modifies network functions for improving the processing of a single attended stimulus. Such modifications could be obtained by reorganizing the network's internal pattern of functional connections by changing the pattern of $\gamma$-band synchronization (Aertsen et al., 1989; König and Schillen, 1991; Singer, 1993; Segev and Rall, 1998; Usrey et al., 2000; Azouz and Gray, 2003; Womelsdorf et al., 2007; Tiesinga and Sejnowski, 2010; Battaglia et al., 2012; Engel et al., 2013).

Based on these considerations, we hypothesized that effective processing of an attended stimulus requires a specific configuration of local neuronal networks in visual cortex. This attention-dependent and stimulus-specific configuration of a local network is established by a specific pattern of $\gamma$-band synchronization between neurons processing the attended stimulus. From this hypothesis, we derive three simple predictions: (1) In the presence of multiple stimuli in the population receptive field ( $\mathrm{pRF}$ ) of a local network, the pattern of $\gamma$-band synchronization and hence, the corresponding configuration of functional connections should depend on the attended stimulus. (2) If this pattern would indeed be stimulus specific, it should be very similar to the pattern observed, if only the attended stimulus is present. (3) Deviating patterns of $\gamma$-band synchronization should reflect erroneous network configurations and go along with deteriorated behavioral performance.

To test the hypothesis, we recorded neuronal activity in area V4, using two closely spaced microelectrodes, while monkeys attended one of two stimuli within the RF. The stimuli were placed within the V4 pRF such that they induced local synchronization and responses of different strength. We found that switching attention between these two stimuli resulted in local $\gamma$-band synchronization strength almost as if the attended stimulus would be present without nearby distractor. Behavioral errors were preceded by local synchronization deviating strongly from that observed for successfully executed trials, while spiking activity showed only small differences between successful and wrong task execution.

\section{MATERIALS AND METHODS}

\section{Surgical Preparation}

Two male macaque monkeys (Macaca mulatta) were implanted under aseptic conditions with a titanium head holder and a recording chamber above area V4. The target area was identified by evaluation of MRI-scans performed before surgery. All procedures were approved by the local authorities (Der Senator für Gesundheit, Bremen, Germany) and were in accordance with the regulation for the welfare of experimental animals issued by the Federal Government of Germany and with the guidelines of the European Union (2010/63/EU) for care and use of laboratory animals.

\section{Behavioral Task}

The animals performed a highly attention-demanding shape-tracking task. In the following, for task parameters differing between individuals, the parameters for monkey $\mathrm{T}$ are mentioned in the text and those for monkey B follow in brackets. Visual stimuli were presented on a 20-inch CRT-monitor with a resolution of 1024 $\times 768$ pixels $(1152 \times 864$ pixels $)$ and a refresh rate of $100 \mathrm{~Hz}$. The screen was placed $90.5 \mathrm{~cm}(92 \mathrm{~cm})$ in front of the monkey that was sitting in a custom-made primate chair. Visual stimulation comprised a fixation point and up to four simultaneously presented complex shapes (Figure 1A). Figure $1 \mathrm{~B}$ shows the sequence of stimuli and events of a single trial: It starts with the appearance of a spatial cue, which indicates the position of the behaviorally relevant stimulus in the upcoming trial. During this period of the trial animals were allowed to move their eyes freely. The spatial cue consisted of a $1^{\circ}\left(1.5^{\circ}\right)$ diameter ring with a linewidth of $0.04^{\circ}\left(0.075^{\circ}\right)$ 
centered over the position of the upcoming target stimulus. For monkey $\mathrm{B}$, the cue contained in addition the initial shape of the upcoming trial, because it helped to increase performance during training of the task. After $2.0 \mathrm{~s}(2.5 \mathrm{~s})$ a central $0.15^{\circ} \times$ $0.15^{\circ}$ fixation point (FP) appeared, which required the animals to start fixation and subsequently to initiate the trial by pressing a lever inside the primate chair within $4.5 \mathrm{~s}$ ( $2.5 \mathrm{~s})$. Following trial start, the spatial cue disappeared (faded within $200 \mathrm{~ms}$ ) and a baseline period of $1050 \mathrm{~ms}$ (1000 ms) began. Subsequently the static presentation period started with the appearance of three or four differently shaped stimuli, all at the same eccentricity between $2.5^{\circ}$ and $3.5^{\circ}\left(2.1^{\circ}-2.5^{\circ}\right)$ of visual angle (Figure 1A). Either one or two adjacent stimuli were located in the lower visual field quadrant contralateral to the recording sites in area V4. The other two stimuli appeared at positions mirrored across the FP in the upper, ipsilateral visual field quadrant. The stimuli presented at each of the four positions differed in color (red, green, yellow, blue; luminance: $3.7-5 \mathrm{~cd} / \mathrm{m}^{2}$, background luminance: $0.03 \mathrm{~cd} / \mathrm{m}^{2}$ ). The assignment of these colors to the four stimulus positions was constant throughout a recording session. Stimuli at all locations could serve as target. The initial complex shapes at each stimulus location were presented statically for $510 \mathrm{~ms}(500 \mathrm{~ms})$ and subsequently started to morph continuously into other complex shapes (see also: Taylor et al., 2005; Grothe et al., 2012). A single morphing cycle (MC), i.e., morphing completely from one shape into another shape, lasted $800 \mathrm{~ms}(1,000 \mathrm{~ms})$. Trials consisted of two to four MCs. All shapes were taken randomly with equal probability out of a set of 8 shapes (6 shapes). The reappearance of the initial shape at the cued stimulus location required the monkeys to release the lever within a time window ranging from $310 \mathrm{~ms}$ before the shapes' complete reappearance to $400 \mathrm{~ms}$ afterwards $(-350 \mathrm{~ms}$ to $150 \mathrm{~ms}$; Figure 1B, dashed rectangle). The appearance of the targets' initial shape within the sequence of MCs at the distractor locations or the distractors own initial shape had to be ignored. For monkey T, all 8 shapes could become initial shape, whereas for monkey $\mathrm{B}$, the initial shape of the target stimulus was always the same, within and across sessions. Throughout the whole trial, the eye position was monitored by video-oculography (monkey T: I Scan Inc., Woburn, MA, USA; monkey B: custom-made eye tracking system) and the direction of gaze was not allowed to deviate from the FP by more than $0.5^{\circ}$. If monkeys released the lever within the response window, they were rewarded with a small amount of diluted fruit juice. If they broke fixation or responded outside the response window, trials were aborted without reward.

\section{Recording Procedure}

Simultaneous intracortical recordings in the upper layers of visual area V4 were performed using two epoxy-insulated tungsten microelectrodes (1-3 M $\Omega$, shank diameter $125 \mu \mathrm{m}$ FHC Inc., Bowdoin, ME, USA; $330 \mu \mathrm{m}$ distance between shanks). The electrode signals were amplified 4000x (1000x) (monkey T: $4 \mathrm{x}$ by a wideband preamplifier MPA32I and $1000 \mathrm{x}$ by a PGA 64, 1-5000 Hz, both Multi Channel Systems GmbH, Germany; monkey B: same setup but gain factor 10 for preamplifier and 100 for PGA) and digitized with $25 \mathrm{kHz}$ sampling rate and
12 bit (16 bit) ADC resolution. The reference electrode for monkey $\mathrm{T}$ was the recording chamber, a titanium cylinder of $25 \mathrm{~mm}$ diameter implanted into the bone and touching the dura. The electrode signals of monkey $\mathrm{B}$ were referenced to a low impedance electrode $(<0.1 \mathrm{M} \Omega)$, positioned on top of an epidural array (contacting the bone), placed above area V1. Before recordings, the $\mathrm{pRF}$ for each recording site was mapped manually as the minimal response field based on multi-unitand LFP-responses, while the animals performed a fixation task. Both microelectrodes were placed such that the recorded neurons shared major parts of their pRFs. Locations and colors for the two stimuli within the overlapping pRFs were chosen such that they caused responses of different strength.

\section{Data Analysis}

Customized scripts for Matlab (version R2013a, MathWorks, Natick, MA, USA) were used for all offline data analysis procedures described below. Data were analyzed for the spiking activity of a small group of neurons by calculating the entire spiking activity (ESA). As a measure for the strength of functional coupling, we calculated the $\mathrm{PhC}$ between two signals, either representing the overall local population activity (LFP) and the activity of a small group of neurons (ESA-LFP PhC) or the activity of two separate groups of neurons of the same local network (ESA-ESA-PhC). For analysis of spiking activity, we used the ESA-signal because it is more sensitive in detecting neuronal responses in data with low signal to noise ratio, since it does not reject sub-threshold events. Furthermore, the independence from thresholding provides the advantage of integrating over all spikes (even small ones) of a population, resulting in a more complete estimate of the actual population response. The ESA of neurons near the recording electrode's tip $(50 \mu \mathrm{m}$ radius according to Brosch et al., 1997) was obtained by band-passing the raw signal using a FIR-filter between 0.3 and $12.2 \mathrm{kHz}$ in forward and backward direction (to avoid phase shifts). Subsequently, the band-limited signal containing the spiking activity was fullwave rectified and low-pass filtered (forward and backward) at $160 \mathrm{~Hz}$ and down-sampled to $1 \mathrm{kHz}$ (Legatt et al., 1980; Frien et al., 2000). As opposed to standard multi-unit activity, this procedure delivers a continuous instead of a binary signal, which is known to represent the spiking activity of multiple neurons surrounding the electrode tip (Supér and Roelfsema, 2005). ESA-responses were obtained by subtracting for each recording site the mean spontaneous activity from the ESAvalues recorded during the analysis period. Spontaneous activity was estimated as the average ESA taken from $150 \mathrm{~ms}$ ( $250 \mathrm{~ms}$ ) after the baseline period started to its end over all correctly performed trials of a recording session. Note, that during baseline period (Figure 1B) no visual stimulus but the FP was present on screen. The LFP was obtained from the recorded signal by lowpass filtering with a FIR-filter $(-3 \mathrm{~dB}$ point at $170 \mathrm{~Hz})$ in forward and backward direction and subsequent down-sampling to $1 \mathrm{kHz}$.

The time-frequency decomposition of ESA and LFP signals was performed by convolving the signals with complex Morlet's wavelets $\omega\left(t, f_{0}\right)=A \exp \left(-t^{2} / 2 \sigma_{t}^{2}\right) \exp \left(2 i \pi f_{0} t\right)$, with $\sigma_{f}=\frac{1}{2 \pi \sigma_{t}}$. Morlet's wavelets have a Gaussian shape both in time (SD: $\sigma_{t}$ ) 
A
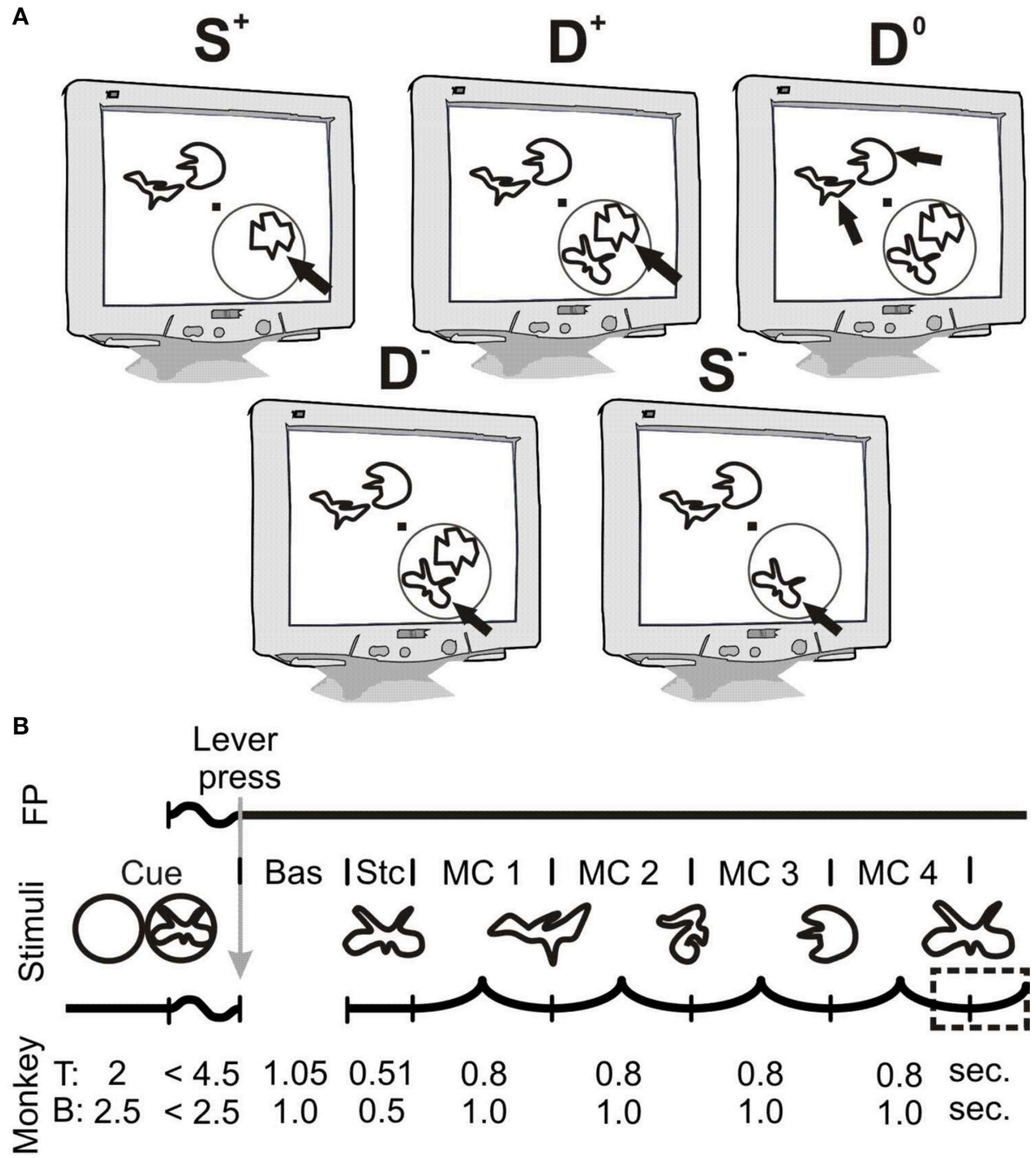

FIGURE 1 | Stimulus configuration and temporal structure of the shape-tracking task. (A) Schematic illustration of task conditions. Black arrows indicate the cued target stimulus; other stimuli serve as distractors. The RF of the recording site in V4 is depicted as a gray circle, which does not appear on the display. Trials could contain either one or two stimuli in the V4 RF. The abbreviations indicate the number of stimuli within the RF ("S" for single and "D" for double presentation in the V4 RF) and the direction of attention to either the stimulus inducing higher "+" or lower values "-" of spiking activity or $\gamma$-synchronization. The condition with two stimuli in the RF but attention directed to stimuli in the opposite hemi-field is referred to as $D^{0}$ (Double Attend Out). (B) Temporal structure of the shape-tracking task. During the cueing period, a spatial cue was present on screen. The cue was a simple ring for monkey T and a ring enclosing the upcoming target shape for monkey B. After appearance of the fixation point (FP), monkeys started fixation and initiated the trial by pressing a lever. Simultaneously, the spatial cue disappeared (faded within $200 \mathrm{~ms}$ for monkey B). After a baseline period (Bas.), followed by static presentation (Stc.) of the initial shapes at each location, they morphed through a sequence of different shapes until the initial shape at the cued location reappeared. Monkeys had to signal this reappearance within a response period indicated by the dashed rectangle. Bas, baseline period; Cue, Cueing period; FP, fixation point; MC, morph cycle; Stc, static presentation period.

and frequency dimension (SD: $\sigma_{f}$ ) and were normalized such that their total energy was 1 . The normalization factor $A$ was defined as:

$$
A=\left(\sigma_{t} \sqrt{\pi}\right)^{-0.5}
$$

Central frequencies $f_{0}$ of the Morlet's wavelets ranged from 5 to $160 \mathrm{~Hz}$ according to the scheme described by Torrence and Compo (1998) with a ratio of $\frac{f_{0}}{\sigma_{f}}=6$ (Tallon-Baudry et al., 1997; Taylor et al., 2005). The wavelet transform provides complex coefficients $\tilde{x}_{j}^{r}$ for electrode $j$ and trial $r$ at time $t$ and frequency $f$, which can be expressed as their amplitude $A$ and phase $\Phi$ :

$$
\tilde{x}_{j}^{r}(t, f)=A_{j}^{r}(t, f) e^{i \Phi_{j}^{r}(t, f)}
$$

The frequency-dependent power of LFP and ESA-signals was computed by taking the square of the absolute value of the convolution's result and dividing it by the Nyquist frequency $(500 \mathrm{~Hz})$. The phase component for each time and frequency bin was used for estimation of $\mathrm{PhC}$ over $N$ trials, between electrodes 
$j$ and $k$, as follows (see also: Lachaux et al., 1999; Grothe et al., 2012):

$$
\operatorname{PhC}(t, f)=\left|\frac{1}{N} \sum_{r} e^{i \Phi_{j}^{r}(t, f)-i \Phi_{k}^{r}(t, f)}\right|
$$

The $\mathrm{PhC}$ was calculated for each electrode pair, thus delivering one ESA-ESA measure and two ESA-LFP pairs (as both sites of the pair can deliver either the ESA or the LFP) for PhC analyses. ESA and LFP signals were never taken from the same electrode to avoid the possibility that the same spike contributed to ESA and LFP. PhC values were bias-corrected by subtracting the expected value (EV) for the $\mathrm{PhC}$, as estimated from the same number trials $(N)$ with random phase relations (Grothe et al., 2012).

$$
E V(N)=\frac{\sqrt{\pi}}{2 \sqrt{N}}
$$

For the analysis of $\mathrm{PhC}$ and power in the $\gamma$-band $(\gamma-\mathrm{PhC}$, $\gamma$-power), we defined the $\gamma$-bands for each animal and measure separately. For this purpose, we computed power and $\mathrm{PhC}$ spectra in the period of MCs 2 and 3. The power spectra of each recording site were normalized by the mean power spectrum obtained during the baseline period of all trials in all conditions of that respective recording site, by first subtraction and then division by the baseline period's spectrum. Subsequently we averaged the normalized power spectra and PhC-spectra of all recording sessions and experimental conditions. The extent of the $\gamma$-band was determined based on the full width at half maximum of the mean $\mathrm{PhC}$ - and power-spectra respectively (Figure 2D, highlighted in gray).

The analysis of behavioral errors required pooling of trials across sessions. This excluded $\mathrm{PhC}$ as measure for synchronization since $\mathrm{PhC}$ requires a constant preferred phase difference across trials. This requirement might not be fulfilled for trials coming from different recording sessions. Therefore we used the magnitude squared coherence (MSC, see also: Carter et al., 1973) which can be computed within single trials and is subsequently averaged over trials. To obtain the MSC we first calculated the complex coherence values $\gamma_{j k}(t, f)$ between the signals of electrodes $j$ and $k$ at each time $(t)$ and frequency $(f)$ bin. This is achieved by multiplying for each trial the complex wavelet coefficient $\tilde{x}_{j}^{r}(t, f)$ with the complex conjugate of $\tilde{x}_{k}^{r}(t, f)$ to obtain the cross spectral density and normalizing it to the square root of the product of their auto spectral densities (again calculated by multiplying the complex wavelet coefficients with complex conjugates, but here of the same signal; complex conjugates are indicated by an overbar):

$$
\gamma_{j k}(t, f, r)=\frac{\tilde{x}_{j}^{r}(t, f) * \overline{\tilde{x}_{k}^{r}(t, f)}}{\left.\sqrt{\left(\tilde{x}_{j}^{r}(t, f) * \overline{\tilde{x}}_{j}^{r}(t, f)\right)\left(\tilde{x}_{k}^{r}(t, f) * \overline{\tilde{x}}_{k}^{r}(t, f)\right.}\right)} .
$$

Subsequently, the absolute value of the complex coherence $\gamma_{j k}(t, f)$ is squared (Carter et al., 1973):

$$
\operatorname{MSC}(t, f, n)=\left|\gamma_{j k}(t, f, n)\right|^{2} .
$$

MSC-values were then averaged over trials. The time and frequency resolved MSC was bias-corrected by subtracting corresponding values derived from a shuffle predictor (Perkel et al., 1967; Gail et al., 2000). This shuffle predictor was obtained by computing 1000 times the MSC between ESA and LFP from randomly shuffled trials and averaging the results for each frequency bin. For computation of the MSC in the $\gamma$-band $(\gamma-$ MSC) we defined a $\gamma$-band based on MSC spectra by the same procedure as described above for the $\gamma$ - $\mathrm{PhC}$.

\section{Experimental Design and Statistical Analysis}

This study includes physiological and behavioral data of two adult male macaque monkeys. The concept of the study demands two stimuli within the same pRF, each of them evoking a specific configuration of the local neuronal network in V4. As quickly accessible indicators for such a difference during the experimental sessions, we used response strength in firing rate and LFPs. We arranged position and color of the individual stimuli within the V4 pRF such that the response strengths were clearly different. To characterize the local processing of different behaviorally relevant stimuli with and without distractor within the V4 pRF, the task paradigm comprises five different conditions.

The two conditions with only one of the two possible stimuli presented inside the $\mathrm{pRF}$ are indicated by an " $\mathrm{S}$ " for single stimulus presentation, whereas the other conditions with both stimuli present are indicated by a " $D$ " for double stimulus presentation. In the double conditions, each of the two stimuli could serve as the target of attention. In order to investigate whether processing of a relevant stimulus implies the same network configuration in absence as well as presence of a competing distractor, we required also in the single conditions attention to the stimulus within the pRF. This ensures a demand to configure a network for processing the same stimulus in corresponding single and double conditions.

Single and double conditions were labeled by a "+" or a "-" sign, depending on the stimulus in the pRF that was attended. Separately for each of the three measures (ESA-responses, ESALFP $\gamma$-PhC, and ESA-ESA $\gamma$-PhC) the labels "+" and "-" were assigned to the stimuli evoking the higher respectively lower values when presented alone. Thus, a stimulus inducing the stronger ESA-responses did not always also induce the higher $\gamma$-PhC (i.e., the same stimulus could be labeled " + " for ESAresponses and "-" for $\gamma$-PhC). For comparison of our ESA results to earlier work we also included a double condition with attention directed away from the pRF (D0). Together this results in five attentional conditions: $\mathrm{S}^{+}, \mathrm{S}^{-}, \mathrm{D}^{+}, \mathrm{D}^{-}$, and $\mathrm{D}^{0}$.

The rationale of the study required that the individual recording sites or site pairs had to fulfill the following criteria to be included in the analysis: (1) Recording sites had to be located in the upper cortical layers. (2) Neurons needed to respond significantly to each of the stimuli presented alone within the pRF, as measured by ESA-response for measures including ESA and by $\gamma$-LFP power for measures including LFP. (3) The values of ESAresponses or $\gamma$-PhC (depending on the analysis) for the two single 

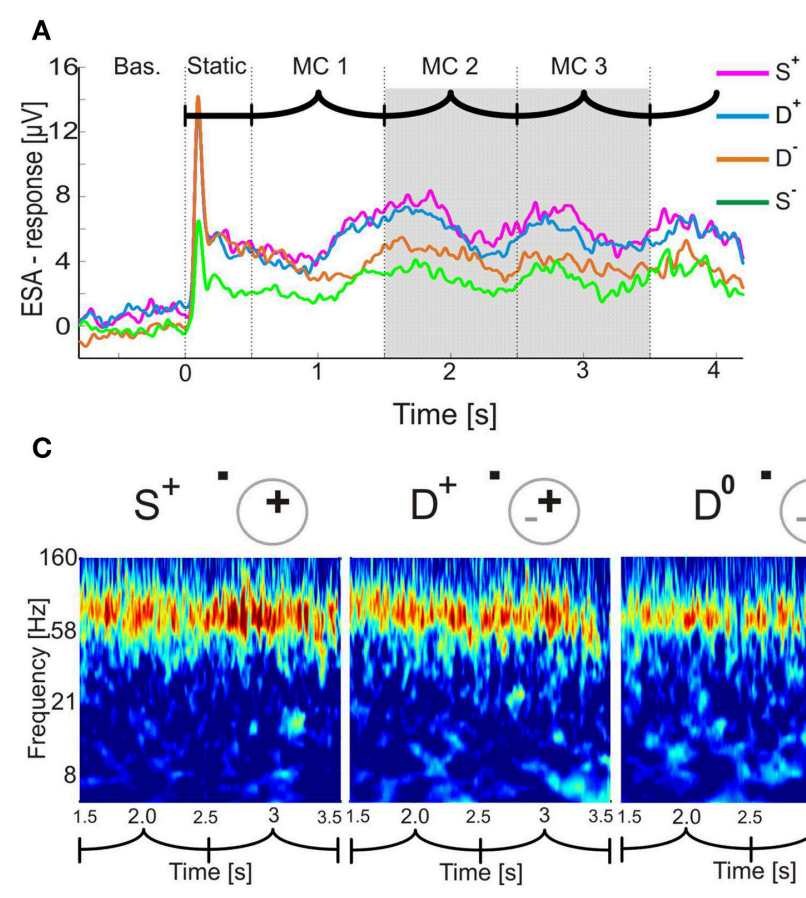

D

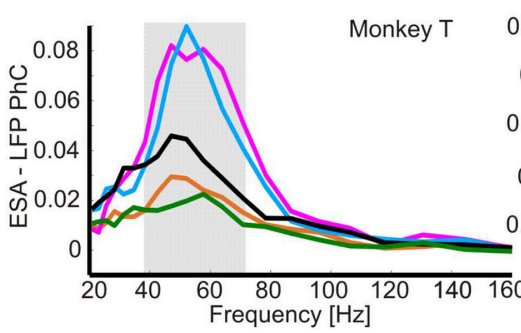

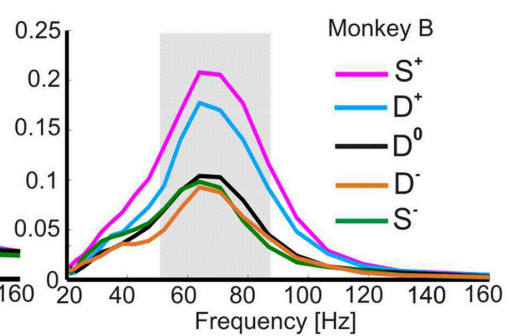

B
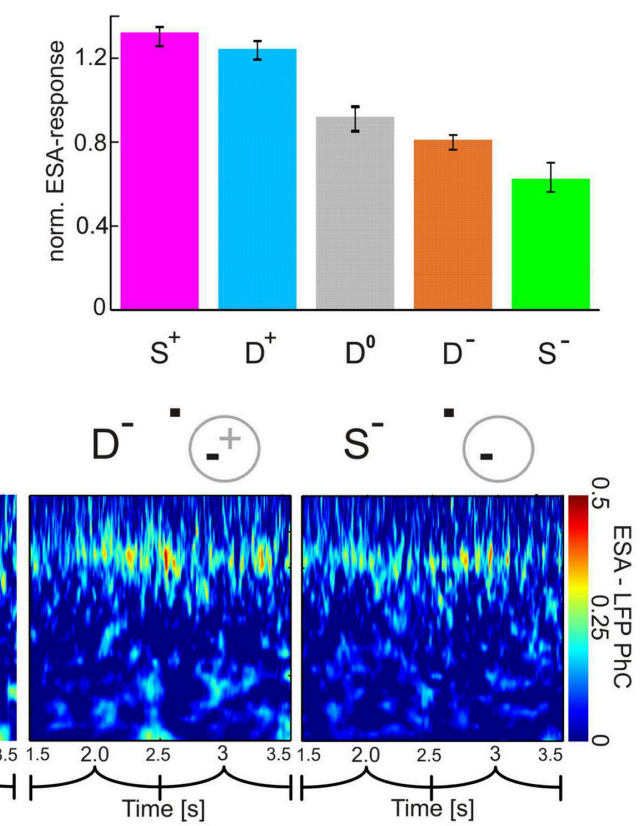

E

FIGURE 2 | Attention-dependent modulation of neuronal responses and their coupling to the local population activity. (A) Time course of mean ESA-responses during the shape-tracking task of an example case (visual stimulation sequence indicated above as in Figure 1). For clarity, the $D^{0}$-condition is omitted. (B) Histogram of normalized median ESA-responses during MC 2 and 3 (highlighted gray in A) and pooled over both animals. Error-bars indicate $95 \%$ confidence intervals. (C) Time-frequency plots of the PhC between ESA and LFP for all task conditions during MC 2 and 3 of an example case. Pictograms indicate stimulus configuration within the V4 pRF. Plus and minus signs depict the stimuli inducing stronger and weaker $\gamma$-PhC, respectively. Bold highlighted signs correspond to the attended stimulus. (D) Median spectra for ESA-LFP PhC of both animals. The individual $\gamma$-bands are highlighted in gray. (E) Scatter-plot of normalized ESA-LFP PhC (median over MC 2/3 and the individual $\gamma$-band) for conditions without vs. with distractor in pRF.

stimulus conditions had to be sufficiently different to make sure the stimuli were driving the local population differently.

Criterion 1) was applied to avoid comparing of phase relations between neurons and overall population activity of different layers. Due to the typical recording procedure (lowering the electrode only until the first responses were found), this meant that recordings happened most likely in the upper layers. The location in the upper cortical layers was verified by the polarity of the evoked potential caused by stimulus onset (Schroeder et al., 1991). The significant activation (criterion 2) of neurons during the analysis window (MC 2/3, for explanation see below) was tested for ESA-values or LFP $\gamma$-power (depending on which measure was used for $\mathrm{PhC}$ estimation) against the respective values during the baseline period (starting after $150 \mathrm{~ms}$ (250 ms for monkey B) to its end; Wilcoxon signed-rank test, critical $\alpha$-level: 0.05).

A sufficient difference of values during single conditions (criterion 3) was required for evaluating whether the ESAresponse or $\gamma$-PhC observed in a double condition is more similar to the corresponding value observed in the $\mathrm{S}^{+}$or in the $\mathrm{S}^{-}$ condition. Therefore, only sites or site pairs were considered, in which the analyzed measure differed by at least a factor of 1.33 between the two single conditions.

All analyses (with the exception of the error trial analysis) were performed within a time window comprising MCs 2 and 3 (Figure 2A, gray background). This analysis window was chosen 
because the target shape never appeared in MC 1, and at latest in MC 4. Therefore, attentional demands might be reduced during these periods. If the initial shape reappeared at the cued location at the end of MC 2 or 3, the time window ended $200 \mathrm{~ms}$ prior to the behavioral response. To exclude the potential survival of response related effects (as drescribed by Mirabella et al., 2007), we performed a control analysis with a cutoff period of $350 \mathrm{~ms}$ before the behavioral response. Neither for ESA-responses nor ESA-LFP $\gamma$-PhC we found differences of the sizes of effects or the level of significances. Individual values differed only marginally (on average by around 1\%) as compared to our original values.

For quantitative analysis of the effect of attention on ESAresponses and $\gamma$-PhC across sessions and animals, the mean values for each of the five different attention conditions observed for a recording site or recording site pair were normalized by dividing them by the average of these five values. The statistical analysis was performed using non-parametric Wilcoxon signedrank tests and Wilcoxon rank-sum tests at a critical $\alpha$-level of 0.05 . In case of multiple comparisons, all $p$-values were Bonferroni corrected, except for $p$-values that were already higher than the critical $\alpha$-level of 0.05 before Bonferroni correction.

To investigate potential relations between neuronal activity patterns and behavioral performance, we compared ESAresponses and the synchronization of ESA with the LFP between correctly performed trials and trials terminated by a false alarm during MC 2/3. The selection criteria for contributing recording sites and site pairs were the same as described above. Because of their small number, the false alarm trials were pooled across sessions for each animal separately and MSC instead of $\mathrm{PhC}$ was used as a measure of synchronization (see above). For the analysis, a time window of $400 \mathrm{~ms}$ (monkey B: $500 \mathrm{~ms}$ ) aligned to and ending $200 \mathrm{~ms}$ before the behavioral response was used. Thus, the duration of the time window was equivalent to half of a MCs' duration. For quantifying the differences between false alarms and correctly executed trials, 1000 randomly compiled sets of correctly performed trials were generated. For each set, we randomly selected the same number of correctly performed trials from each session as the session contributed false alarms. From these 1000 sets of correctly performed trials, the distributions of $\gamma$-MSC values were computed within the same time windows as for the corresponding false alarm trials in the corresponding sessions. The pooling procedure and random selection of trials was identical for investigating ESA-responses. Based on these distributions, we estimated the $\mathrm{z}$-score values for $\gamma$-MSC and ESA-responses observed in the false alarm trials in comparison to correctly performed trials and derived corresponding confidence levels (probability derived from $\mathrm{z}$-score chart).

\section{RESULTS}

We investigated attention-dependent changes of local network configuration in visual area V4 of two macaque monkeys, while the animals performed an attention-demanding shape tracking task (Taylor et al., 2005; Grothe et al., 2012). Briefly, animals had to covertly attend one of three or four stimuli with different shapes, colors and luminance, of which one was previously cued (Figure 1A). After static presentation, the shapes of all stimuli started morphing into other, randomly selected shapes, color, and luminance did not change (Figure 1B). Trials could contain up to four such MCs. Animals were required to detect the reappearance of the initial shape at the cued location. Animals broke fixation in $5.5 \%$ (monkey $\mathrm{T}$ ) and $24.9 \%$ (monkey B) of trials respectively, the average performance disregarding fixation errors was 87.1 and $93.3 \%$ correct trials for monkey $\mathrm{T}$ (28 recording sessions) and B (34 recording sessions), respectively. In $6.6 \%$ (monkey $\mathrm{T}$ ) and $3.8 \%$ (monkey $\mathrm{B}$ ) of all trials (across all stimulus conditions), the response occurred before reappearance of the initial shape (false alarms), and in 6.3\% (monkey $\mathrm{T}$ ) and $2.9 \%$ (monkey B) the response occurred too late (misses). For trials with attention directed to a stimulus inside the $\mathrm{pRF}$, the proportion of false alarms differed significantly for one animal between trials with one and two stimuli in the pRF (monkey T: with distractor in pRF $6.8 \%$, without distractor $5.1 \%, \mathrm{z}=2.5504$, $p=0.0108$; monkey $\mathrm{B}$ : with distractor in $\mathrm{pRF} 2.0 \%$, without distractor $2.2 \%, \mathrm{z}=-0.561, p=0.5748$; Wilcoxon signed-rank test).

To investigate attention-dependent modulations of $\gamma$-PhC and ESA-responses within local V4 networks, we recorded simultaneously with two closely spaced microelectrodes ( $330 \mu \mathrm{m}$ distance between shanks) from 118 recording sites in supragranular layers 2/3 (monkey T: 54 , monkey B: 64). Data were gathered in 62 recording sessions (monkey T: 28, monkey B: 34). Based on manual mapping, we found 57 pairs with overlapping pRFs (monkey T: 26, monkey B: 31).

\section{Attention-Dependent Modulation of Spiking Activity}

We first verified whether ESA-responses in our paradigm revealed a similar pattern of attentional modulation as described in previous studies based on single-unit firing rates (Moran and Desimone, 1985; Treue and Maunsell, 1996; Luck et al., 1997; Desimone, 1998; Reynolds et al., 1999; Treue and Martínez Trujillo, 1999; Lee and Maunsell, 2010; Ni et al., 2012). These studies showed consistently that when two stimuli are present in a RF, firing rates were modulated by attention to similar levels as for the attended stimulus presented alone. Without attention, firing rates were intermediate. Figure $2 \mathrm{~A}$ shows an example for ESA-responses under different attentional conditions with either one $\left(\mathrm{S}^{+} / \mathrm{S}^{-}\right)$or two $\left(\mathrm{D}^{+} / \mathrm{D}^{-}\right)$stimuli inside the same pRF (cf. Methods for details of labeling task conditions). Due to our study design, ESA-responses were stronger during $\mathrm{S}^{+}$ conditions than during $\mathrm{S}^{-}$conditions. When both stimuli were present in the pRF, the ESA-responses were in-between those for the single stimulus conditions and depended on the allocation of spatial attention. ESA-responses were stronger when the well activating stimulus was attended $\left(\mathrm{D}^{+}\right)$than when the less activating stimulus was attended $\left(\mathrm{D}^{-}\right)$. Quantitative analysis of 80 recording sites (monkey T: 37, monkey B: 43) revealed significant differences between the two conditions with both stimuli present in the pRF (Figure 2B). ESA-responses 
were significantly larger during $\mathrm{D}^{+}$(median 1.3) than during $\mathrm{D}^{-}$conditions (median: $0.8 ; \mathrm{z}=7.293$, Bonferroni-corrected $p<10^{-11}$, Wilcoxon signed-rank test). Thus, ESA-responses during double conditions were shifted toward the response levels induced when the attended stimulus was presented in isolation $\left(\mathrm{S}^{+}\right.$: median: 1.3 ; $\mathrm{S}^{-}$: median: 0.6$)$. Conditions which required the animals to direct attention away from the two stimuli inside the pRF to one of the stimuli located in the opposite hemi-field resulted in intermediate responses $\left(\mathrm{D}^{0}\right.$ : median 0.9$)$. These responses were significantly different from the responses for the two other conditions with two stimuli in the pRF $\left(\mathrm{D}^{+} / \mathrm{D}^{0}: \mathrm{z}=7.77, p<10^{-10} ; \mathrm{D}^{-} / \mathrm{D}^{0}: z=-3.0504, p=\right.$ 0.0069 ; $p$-values are Bonferroni corrected, Wilcoxon signed-rank test).

To quantify the extent of attentional modulation of responses in the double conditions and relate it to the difference between the responses caused by each of the two different stimuli alone we used an attentional modulation index (AMI). It is computed as the ratio of the attention-dependent difference between responses obtained in the $\mathrm{D}^{+}$and $\mathrm{D}^{-}$condition to the difference between responses in the $\mathrm{S}^{+}$and $\mathrm{S}^{-}$condition $\left(\mathrm{AMI}=\left(\left(\mathrm{D}^{+}-\mathrm{D}^{-}\right) /\right.\right.$ $\left.\left.\left(S^{+}-S^{-}\right)\right)^{*} 100\right)$. The AMI reaches $100 \%$, if attention modulated ESA-values such that the differences between double conditions on the one side and single conditions on the other side are equal. An AMI of zero indicates that there is no effect of attention when both stimuli are present. AMI values larger than $100 \%$ indicate that the difference of responses between $\mathrm{D}^{+}$and $\mathrm{D}^{-}$ is even larger than between the single conditions. Negative values correspond to an opposite modulation in the double conditions as compared to the single conditions $\left(\mathrm{D}^{+}\right.$smaller than $\mathrm{D}^{-}$). For ESA-responses the median AMI value of $68.4 \%$ shows that the difference between $\mathrm{D}^{+}$and $\mathrm{D}^{-}$conditions is similar to that observed during the respective single conditions, but does not reach the same size. In summary, the attentiondependent modulation of ESA-responses under the stimulus and task conditions of the present experiments is well in line with previous findings on single-unit firing rates in areas V4 and MT.

\section{Attention-Dependent Changes of $\gamma$-PhC Between Neurons and Overall Population Activity}

After having confirmed the expected effect of selective attention on response strength, we tested our hypothesis that effective processing of an attended stimulus is associated with a specific configuration of functional connectivity within the local neuronal network. To this end, we compared the strength of $\gamma$ band synchronization between a small group of neurons (ESA) and the overall population activity (as measured by the LFP) either for a particular stimulus presented alone or together with a distractor in the pRF.

We calculated the PhC between ESA and LFP taken from two separate, closely spaced electrodes. In the following ESA-LFP $\mathrm{PhC}$ analysis, the designation of stimuli as “+” or “-” depended on the strength of $\gamma$-PhC induced by the two stimuli when presented alone. Figure 2C provides an example case of monkey
$\mathrm{B}$, showing the phase coupling between ESA and LFP in the $\gamma$ band $(57.7 \mathrm{~Hz}$ to $86.8 \mathrm{~Hz})$, persisting throughout MCs 2 and 3 . The time averaged strength of the $\gamma$-PhC for the two conditions with only one stimulus in the pRF was 0.34 for the $\mathrm{S}^{+}$and 0.13 for the $S^{-}$condition, indicating two configuration states that are separable with our network interaction proxy. When both stimuli were simultaneously present in the pRF and one of them was attended, the degree of synchronization closely matched the values of the corresponding singe stimulus condition $\left(\mathrm{D}^{+}\right.$: mean $0.3 ; \mathrm{D}^{-}:$.mean 0.15$)$. With attention directed outside the $\mathrm{pRF}$, the $\gamma$-PhC was intermediate $\left(D^{0}\right.$ : mean 0.26$)$.

Mean PhC-spectra for all recording site pairs confirmed the similarity of $\gamma$-PhC between conditions requiring to attend the same stimulus either in the presence or in absence of a distractor inside the pRF, for both animals (Figure 2D). A stimulus inducing low $\gamma$ - $\mathrm{PhC}$ values when presented alone $\left(\mathrm{S}^{-}\right)$induced similarly low $\gamma$ - $\mathrm{PhC}$ values even in the presence of a distractor inducing high $\gamma$ - $\mathrm{PhC}$ when presented alone $\left(\mathrm{D}^{-}\right)$. Conversely, when the stimulus inducing strong $\gamma$-PhC was attended, the level of $\gamma$-PhC stayed similarly high when a distractor inducing weak $\gamma$-PhC was present in the pRF (compare $\mathrm{S}^{+}$and $\mathrm{D}^{+}$). The attention-dependent modulation of $\gamma$-PhC for all ESA-LFP pairs is shown in Figure 2E. The scattering of entries around the diagonal line indicates the similarity between the normalized $\gamma$-PhC values for attending a stimulus without versus with distractor in the pRF for both animals $(n=90)$. Note, that each recording site pair may deliver two ESA-LFP pairs since each electrode contributed an ESA and a LFP signal. The differences between normalized $\gamma$-PhCvalues for the two conditions requiring to attend the stimulus inducing strong $\gamma$-PhC were small but significant $\left(\mathrm{S}^{+}\right.$: median 1.42, $\mathrm{D}^{+}$: median 1.27, $z=4.6296$, Bonferroni corrected $p<$ $10^{-4}$, Wilcoxon signed-rank test). For conditions requiring to attend the stimulus inducing weak $\gamma$-PhC, the difference was not significant $\left(\mathrm{S}^{-}\right.$: median $0.68, \mathrm{D}^{-}$: median $0.63 ; z=1.3017, p=$ $0.1930)$. In contrast, the difference between double conditions was large and highly significant $\left(\mathrm{D}^{+}\right.$: median $1.27, \mathrm{D}^{-}$: median: $0.63, z=7.1481$, Bonferroni corrected $p<10^{-11}$, Wilcoxon signed-rank test). The AMI, as a measure for the degree of attentional modulation in the double conditions as compared to the single conditions reached a median value of $81.8 \%$ and was significantly larger than the AMI for ESA-responses (ESAresponses: $68.4 \%, p=0.0142, z=-2.4523$, Wilcoxon ranksum test). This close match of $\gamma$-PhC between ESA and LFP signals for conditions requiring to attend the same stimulus $\left(\mathrm{S}^{+} / \mathrm{D}^{+}\right.$and $\left.\mathrm{S}^{-} / \mathrm{D}^{-}\right)$also holds true for the individual animals (see Table 1). We found these significant modulations of $\mathrm{PhC}$ only in the $\gamma$-frequency range, but not for other frequency bands.

To ensure that the stimulus specific differences of $\gamma$ PhC do not reflect very weak or lacking ESA and LFP oscillations, we examined the strength of underlying $\gamma$ oscillations. Supplementary Figures 1A and B show that not only during $\mathrm{S}^{+}$and $\mathrm{D}^{+}$conditions, but also during $\mathrm{S}^{-}$and $\mathrm{D}^{-}$conditions, $\gamma$-band oscillations were sufficiently large to ensure meaningful phase estimations for the $\mathrm{PhC}$-measure. To investigate whether the modulations of $\gamma$-PhC simply 
TABLE 1 | Comparison of ESA-LFP $\gamma$-PhC values observed during different stimulus conditions for both animals.

\begin{tabular}{|l|c|c|c|c|}
\hline $\begin{array}{l}\text { Compared } \\
\text { conditions }\end{array}$ & \multicolumn{2}{|c|}{$\begin{array}{c}\text { Monkey T } \\
(\boldsymbol{n}=34)\end{array}$} & \multicolumn{2}{c|}{$\begin{array}{c}\text { Monkey B } \\
(\boldsymbol{n}=\mathbf{5 6})\end{array}$} \\
\hline $\mathrm{S}^{+} / \mathrm{D}^{+}$ & $1.48 / 1.54$ & $p=0.4675$, & $1.41 / 1.18$ & $p<10^{-7 \star}$, \\
& & $\mathrm{z}=0.7266$ & & $\mathrm{z}=5.4408$ \\
\hline $\mathrm{D}^{+} / \mathrm{D}^{-}$ & $1.54 / 0.55$ & $p<10^{-3 *}$, & $1.18 / 0.63$ & $p<10^{-8 *}$, \\
& & $\mathrm{z}=4.0433$ & & $\mathrm{z}=5.9791$ \\
\hline $\mathrm{D}^{-} / \mathrm{S}^{-}$ & $0.55 / 0.51$ & $P=0.1909$, & $0.63 / 0.78$ & $p=0.0027^{\star}$, \\
& & $\mathrm{z}=1.3079$ & & $\mathrm{z}=3.3199$ \\
\hline
\end{tabular}

The leftmost column describes the two task conditions that are compared. For each animal, the left column shows the normalized median $\gamma$-PhC values for these two conditions, whereas the right column provides the results of the corresponding Wilcoxon signed-rank tests. The asterisk indicates Bonferroni-corrected p-values. ESA-LFP $\gamma$-PhC values during ST-task for individual animals.

reflect the modulations in ESA and/or LFP $\gamma$-power, we analyzed how well the reduction of $\gamma$-PhC between $\mathrm{D}^{+}$ and $\mathrm{D}^{-}$conditions can be explained by changes in ESA and LFP $\gamma$-power (Supplementary Figures $\mathbf{1 C}, \mathbf{D}$ ). There were no significant correlations between the $\gamma$-PhC reduction and modulations of LFP or ESA $\gamma$-power $(\gamma$-PhC/ $\gamma$-LFP-power: Pearson's correlation, $r=0.1821, p=0.11 ; \gamma$-PhC/ $\gamma$-ESA-power: $r=0.17, p=0.1252$ ).

\section{Attention-Dependent Modulation of Functional Coupling Between Groups of Neurons}

Processing of different stimuli in a local network is thought to depend on different patterns of functional coupling strengths between its neurons. Therefore, we investigated whether the strength of functional connections between two small groups of neurons as measured by the ESA-ESA $\gamma$-PhC in the presence of two stimuli matched that observed for the attended stimulus presented alone. Here the designation of stimulus conditions as $\mathrm{S}^{+}$or $\mathrm{S}^{-}$was based on the strength of the ESA-ESA $\gamma$-PhC induced by the two different stimuli shown in these conditions. Contrary to our expectation, we found that the difference between double conditions $\left(\mathrm{D}^{+} / \mathrm{D}^{-}\right)$was much smaller than between single conditions $\left(\mathrm{S}^{+} / \mathrm{S}^{-}\right)$as reflected by a median AMI of $57.9 \%(n=44)$. This raises the question whether this rather low AMI is characteristic for the $\gamma$-PhC between two subpopulations of neurons of the same population. The distribution of AMI values (Figure 3A) indicates that this is not the case since the AMI differed strongly over a wide range between pairs.

To investigate whether the wide range of AMI-values might be related to different response characteristics of the two constituting sites, we split the pairs according to their AMI value into three equally sized groups. (Figure $\mathbf{3 A}$, dashed lines separate low, medium and high AMI group). First, we analyzed the relation between the pairs' AMI values for $\gamma$-PhC and the ESA-responses of their corresponding sites during $\mathrm{S}^{+}$conditions. Almost half (47\%) of the pairs of the low AMI-group (Figure 3B, red dots) had at least one site, which responded very weakly (ESA response strength $<0.05$, border indicated by gray dashed lines in Figure 3B) to the stimulus inducing high $\gamma$-PhC. There were no such sites for the high AMI-group (blue dots) and only two pairs with such low responses for the medium group (green dots). Chi-square tests of independence confirmed, that there are significantly more such pairs in the low AMI group than in the high AMI-group $\left(\chi^{2}(1, N=29)=8.61\right.$, Bonferronicorrected $p<0.01)$. All other comparisons between groups revealed no significant differences after Bonferroni correction (low AMI/medium AMI; medium AMI/high AMI; $\chi^{2}(1, N=30)$ $<3.8$, Bonferroni-corrected $p>0.05)$. Yet, the low-AMI group seems to consist of two groups of pairs, one with at least one site showing rather low responses $(<0.05)$ and another one where both sites showed normal response levels (values around 0.3-0.6). This may indicate that there are further combinations of response properties of a pair that go along with low AMI values.

Therefore, we analyzed whether the ratio between ESAresponses to the two stimuli might influence the AMI-values of $\gamma$-PhC as well. For each recording site a specificity index (SI) was calculated, which describes the degree of similarity (or dissimilarity) of the responses for the two different stimuli. The index reflects the difference between the ESA-responses during the $\mathrm{S}^{+}$and the $\mathrm{S}^{-}$conditions divided by their sum $\left(\left(S^{+}-S^{-}\right) /\left(S^{+}+S^{-}\right)\right)$. Positive values indicate a preference for the stimulus of the $\mathrm{S}^{+}$condition, negative values for the stimulus of the $\mathrm{S}^{-}$condition and zero the same response strength for both stimuli. The scatterplot in Figure 3C depicts the SI-indices of the two contributing sites for each recording site pair of the three different AMI groups (high, medium and low values). A pair that has at least one site, which responds very similar to both stimuli, would be located between the dashed gray lines (Figure 3C; SI-values between -0.05 and 0.05 ). The entry of a pair with opposite stimulus preferences would be located in the lower right quadrant of the scatterplot (because the site with the higher SI-value is plotted on the X-axis). When comparing the distributions of entries it becomes obvious, that more entries of the low AMI group (red dots) are located between the gray dashed lines than for both other groups. Furthermore, there are no entries of the high AMI-group with opposite stimulus preference. Chi-square tests confirmed that the low AMI-group contained in comparison to the medium and the high AMI-group more pairs where at least one site shows almost no difference in responses to both stimuli (SI-values between -0.05 and 0.05 ) or even opposite stimulus preferences (high/low group: $\chi^{2}(1, N$ $=29)=10.208$, Bonferroni-corrected $p<0.005$; medium $/$ low group: $\chi^{2}(1, N=30)=6.53$, Bonferroni-corrected $\left.p<0.04\right)$. The corresponding differences between high and medium AMI group were not significant $\left[\chi^{2}(1, N=29)=0.68, p=0.41\right]$.

In summary, we found that high AMI-values correlate with a sufficient level of responses of both sites to the attended stimulus (Figure 3B). Furthermore, high AMI-values also correlate with higher levels of stimulus specificity for the same stimulus of the two constituting sites (Figure 3C). Thus, the weak synchronization in the $\mathrm{D}^{+}$condition (Figure $\mathbf{3 A}$, compare insets), which results in low AMI values, is observed in pairs with at least one site almost not responding to the attended stimulus (Figure 3B) or responding equally strong to both stimuli (Figure 3C). Such sites may therefore receive comparatively high proportions of signals related to the non-attended stimulus 
A

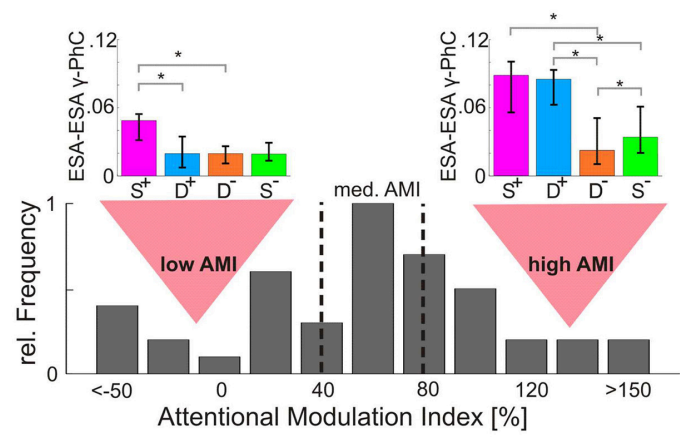

B

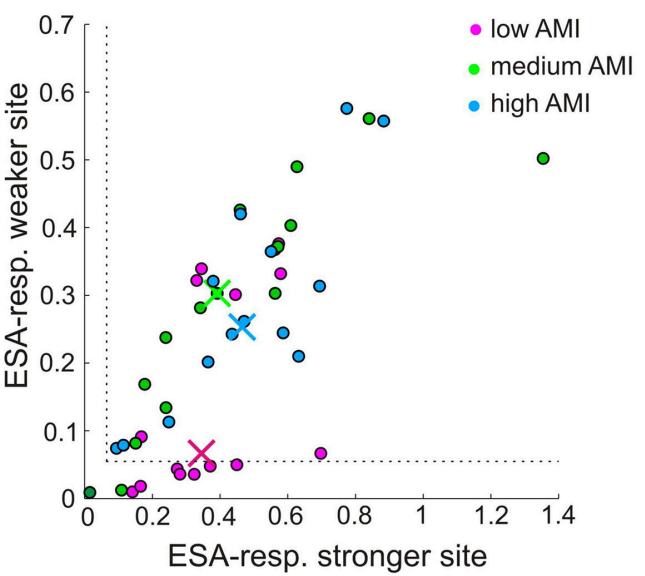

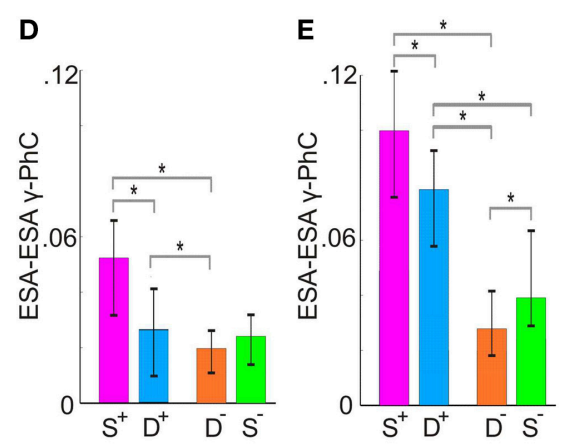

C

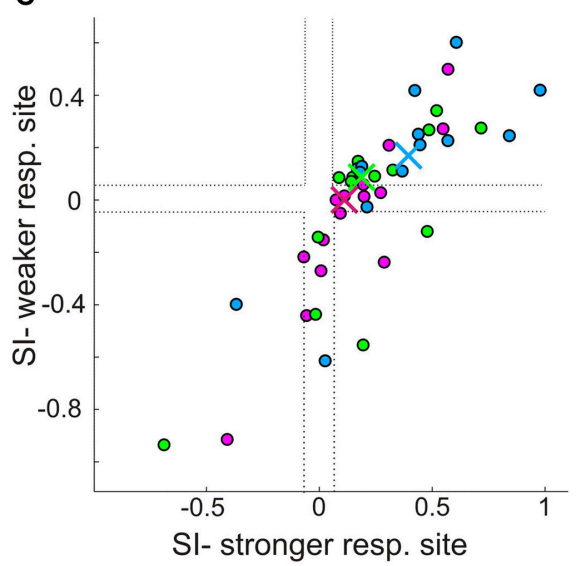

FIGURE 3 | Dependence of ESA-ESA $\gamma$-PhC modulation on level of ESA-responses and stimulus specificity. (A) Distribution of AMI-values based on ESA-ESA $\gamma$-PhC $(n=44)$. The dashed vertical lines indicate borders between the third with lowest, the third with medium, and the third with highest AMl-values. The bar-plot insets above the distribution depict for the low and the high AMl group the median normalized $\gamma$-PhC between ESA-signals, which determine the AMI values. (B) Scatter-plot of normalized ESA-responses for the stimulus inducing higher $\gamma$-PhC between ESA-signals ( ${ }^{+}$condition). An entry represents the ESA-responses of both recording sites contributing to the $\gamma$-PhC, with the site showing higher ESA-responses plotted on the X-axis. The different colors represent the high, medium, and low AMl-group, as illustrated in (A). The median values for each group are depicted as correspondingly colored crosses. (C) Same as in (B), but for the stimulus specificity of the sites constituting a pair. Sites showing higher SI-values are plotted on the X-axis. (D) Histogram of median normalized $\gamma$-PhC between ESA signals of the unspecific group (at least one site with ESA-responses below 0.05, see B or SI-values between -0.05 and 0.05 or with opposite stimulus preference, see (C) cases between dashed lines and in lower right quadrant). The error-bars indicate $95 \%$ confidence intervals. Asterisks indicate significant differences ( $p<0.05)$ (E) same as in D, but for the specific group (pairs which were not classified as unspecific, see $\mathbf{D}$ ).

during the $\mathrm{D}^{+}$condition. Strong synchronization between those neurons with a group of neurons processing mainly the attended stimulus could lead to a mixing of signals from target and distracter stimuli, which would counteract the enhanced and selective processing of the attended stimulus. It might therefore be beneficial that attention does not include those neurons into the ensemble processing the attended stimulus by enhancing their functional coupling.

In order to test whether such relations of response characteristics determine the different effects of attention $\gamma$-PhC, we split the 44 ESA-ESA pairs into two groups: One in which the promotion of distractor-related signals is unlikely and a second where this is more likely. Pairs were assigned to the first group (specific group) if both sites showed a sufficient response of at least 0.05 in the $\mathrm{S}^{+}$condition and a preference for the same stimulus (both SI-values above +0.05 or both below -0.05 ). The remaining pairs were assigned to the second group (unspecific group).
The median $\gamma$-PhC values of the unspecific group $(n=24)$ are shown in Figure 3D, the corresponding $\gamma$-PhC values for the specific group $(n=20)$ in Figure 3E. The most evident difference between both groups is between the median $\gamma$-PhC values during $\mathrm{D}^{+}$-conditions. The unspecific group (Figure 3D) reached with a median value of 0.026 only $49.1 \%$ of the $\gamma$-PhC evoked during $\mathrm{S}^{+}$-conditions (median 0.053). For the specific group (Figure $3 \mathrm{E}$ ), the $\gamma$-PhC during $\mathrm{D}^{+}$-conditions (median 0.08 ) reaches $80.4 \%$ of the value evoked by the $\mathrm{S}^{+}$-condition (median: 0.1). However, for both groups these differences were significant (unspecific group: $p<10^{-3}, z=3.9429$; specific group: $p=0.0015, z=3.6213$, Wilcoxon signed-rank test, all $p$-values are Bonferroni corrected).

Another difference between specific (Figure 3E) and unspecific (Figure 3D) group can be observed when comparing $\mathrm{D}^{+}$and $\mathrm{D}^{-}$conditions. For the specific group, the difference was large and significant $\left(\mathrm{D}^{+}\right.$: median $0.08, \mathrm{D}^{-}$: median 0.027 , Bonferroni corrected $p<10^{-3}, z=3.8826$ ), whereas it was 
small, albeit significant for the unspecific group $\left(\mathrm{D}^{+}:\right.$median $0.026, \mathrm{D}^{-}$: median 0.021, Bonferroni corrected $p=0.036, z=$ 2.6857, both Wilcoxon signed-rank test). The $\gamma$-PhC of the unspecific group recorded during $\mathrm{D}^{+}$conditions (median: 0.026) was not even significantly different from those recorded during $\mathrm{S}^{-}$conditions (median 0.028; $p=0.3758, z=0.8857$, Wilcoxon signed-rank test). Yet, the same conditions evoked highly significant differences for pairs of the specific group $\left(\mathrm{D}^{+}\right.$: median 0.08, $\mathrm{S}^{-}$: median 0.03; Bonferroni corrected $p<10^{-3}, z=3.8453$, Wilcoxon signed-rank test). These differences in $\gamma$-PhC during double conditions explain the large and significant differences in AMI-values between both groups, with a median AMI of $84.7 \%$ for the specific group and only $29.9 \%$ for the unspecific group $\left(p<10^{-5}, z=4.0423\right.$, Wilcoxon signed-rank test). The AMI for the unspecific group was significantly lower than AMI-values for ESA-responses and ESA-LFP $\gamma$-PhC (ESA-responses/unspecific group: $p<10^{-4}$, $z=-4.1392$; unspecific group/ESA-LFP AMI: $p<10^{-10}, z=$ $-6.4883, p$-values were Bonferroni-corrected). AMI-values for the specific group were significantly larger than those of ESA-responses and ESA-LFP $\gamma$-PhC (ESA-responses/specific group: $p=0.032, z=2.4085$; specific group/ESA-LFP AMI: $p<$ $10^{-8}, z=5.9558, p$-values were Bonferroni-corrected).

In summary, the attention-dependent modulation of $\gamma$-PhC between two neuronal sub-populations of the same local network depends on the response characteristics of their neurons for the two stimuli located in the pRF. The modulation is almost identical in conditions with and without distractor if both subpopulations respond sufficiently well to an attended stimulus and share the same stimulus preference. In contrast, if one of the two sub-populations shows only low responses to one of the stimuli, or the sites do not share th567e same stimulus preference, the strong attention-dependent difference between double conditions vanished.

\section{Network State and Behavioral Outcome}

The results so far showed that attention modulates the pattern of $\gamma$-PhC in dependence of the attended stimulus with very similar values of $\gamma$ - $\mathrm{PhC}$ in conditions with and without nearby distractor present. Hence, successful stimulus processing seems to depend on this specific pattern of synchronization within the local V4 network. If this holds true, the question arises, whether unsuccessful behavioral outcomes are associated with an incorrect pattern of $\gamma$-synchronization. Thus, we compared the $\gamma$-synchronization and spiking activity of periods directly preceding a behavioral error. A sufficient number of errors occurring during $\mathrm{MC} 2 / 3$ for $\mathrm{D}^{+}$and $\mathrm{D}^{-}$conditions were available only for false alarms for both animals. Therefore we here show the false alarm trials pooled across all recording sessions for each animal individually. A comparison of misses and correctly executed trials for the monkey with a sufficient number of misses is shown in Supplementary Figure 2. The pooling across sessions made it necessary to investigate $\gamma$-band synchronization between ESA and LFP signals based on magnitude-squared coherence (cf. Materials and Methods for details). We analyzed $\gamma$-synchronization and ESA-responses within a time period of $400 \mathrm{~ms}$ for monkey $\mathrm{T}$ and $500 \mathrm{~ms}$ for monkey B. (Materials and Methods) terminating $200 \mathrm{~ms}$ before the behavioral response.

The normalized ESA-responses were similar between periods preceding false alarms (Figure 4A, light blue and orange bars) and correct responses (Figure $\mathbf{4 A}$, dark blue and orange bars) when attending the stimulus inducing stronger responses $\left(\mathrm{D}^{+}\right)$ as well as for attending the stimulus inducing weaker responses $\left(\mathrm{D}^{-}\right)$. There was no significant difference during $\mathrm{D}^{+}$conditions for monkey $\mathrm{T}$, and only small but significant differences for monkey B (monkey T: hits: 1.26 , errors: $1.12, n=52$, $\mathrm{z}$-score $=$ -1.20 ; $p=0.12$; monkey B: hits: 1.40 , errors: $1.23, n=39$, zscore $=-2.22, p=0.013$; $\mathrm{z}$-transform, cf. section Materials and Methods). Similarly, the differences between ESA-responses in false alarm and correctly executed trials during $\mathrm{D}^{-}$were not significant for both animals (monkey T: hits: 0.53 , errors: 0.50 , $n=108$, $\mathrm{z}$-score $=-0.57$; monkey B: hits: 0.78 errors: $0.80, n=$ $58, \mathrm{z}$-score $=0.26 ; p>0.05$ for both, $\mathrm{z}$-transform).

In contrast to ESA-responses, the $\gamma$-synchronization between ESA and LFP (Figure 4B) showed a strong reduction for false alarm trials. During $\mathrm{D}^{+}$conditions, the $\gamma$-synchronization was more than $98 \%$ weaker for monkey $\mathrm{T}$ and still $62 \%$ weaker for monkey B in periods preceding a behavioral error as compared to correctly performed trials (monkey T: hits: 0.07 , errors: 0.001 , $n=28, z$-value $=-2.5083$; monkey B: hits: 0.08 , errors: 0.03, $n=38, z$-value $=-2.7822 ; p<0.005$ for both). During $\mathrm{D}^{-}$ conditions, the values between false alarm trials and correctly performed trials did not differ significantly (monkey T: hits: 0.02, errors: 0.02, $n=95, z$-value $=-0.0978, p=0.46$; monkey $\mathrm{B}$ : hits: 0.053 , errors: $0.052, n=38, z$-value $=-0.1123, p=0.46)$. In summary, false alarms seem to correlate with a reduced level of $\gamma$ synchronization within the local neuronal network as compared to periods preceding correctly executed trials. In contrast, spiking activity does not reflect the erroneous behavioral responses, indicating the relevance of correctly configured networks for successful behavior.

\section{DISCUSSION}

Within the frame of this work, we examined whether the ability of neuronal networks to perform different functions on the same input could be implemented by an attentiondependent reconfiguration of the local neuronal networks. We hypothesized that this reconfiguration within the local neuronal networks is established by changing the pattern of functional connections between the network's neurons by adjusting the strength of their $\gamma$-synchronization. Thus, for effective processing of an attended stimulus, a stimulus-specific pattern of $\gamma$ synchronization would be necessary and patterns deviating from this specific configuration would result in a decreased stimulus processing.

Indeed, our results show that in the presence of two stimuli in the pRF, the $\gamma$-PhC between the spiking activity and the overall population activity within a local network depends on the attended stimulus and is highly similar to the values observed when the attended stimulus was presented alone in the pRF. The same holds true for $\gamma$-PhC of spiking activity between two groups of neurons of the same local network, but only when both responded sufficiently well to this stimulus and 
A

\section{Monkey $\mathrm{T}$ Monkey B}
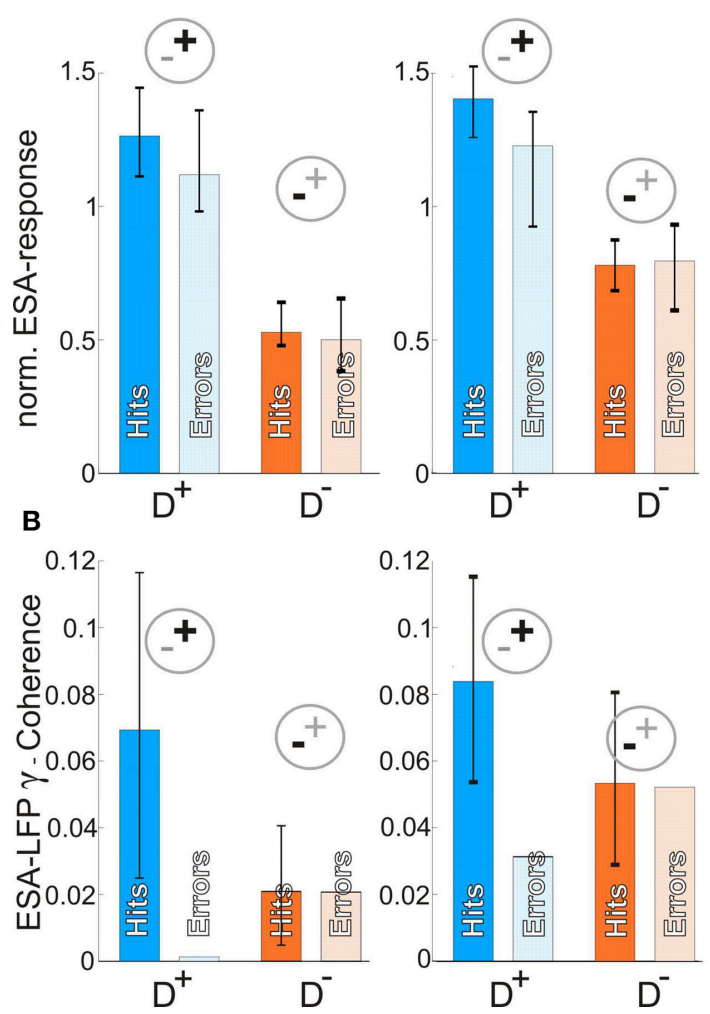

FIGURE 4 | Neuronal correlates of different behavioral outcomes. (A) Median normalized ESA responses just before correct (dark blue and orange) and erroneous (light blue and orange bars) responses in trials requiring attention for one of the two stimuli within the pRF. Error-bars indicate 95\% confidence intervals. (B) $\gamma$-coherence between ESA and LFP for the same conditions as in (A). Note, that in contrast to A error-bars here indicate $95 \%$ of coherence values generated from 1,000 times compiling sets of correctly performed trials (c.f. Materials and Methods). The coherence value for error trials represents the coherence of all error trials pooled (due to small number). The value has to be interpreted with respect to the distribution of correctly performed trials and has no error-bars, since it is only one value. For abreviations and pictograms see

Figures 1, 2.

both shared the same stimulus preference. Correspondingly, the attentional modulation index (AMI) for ESA-LFP $\gamma$-PhC and ESA-ESA $\gamma$-PhC reached 82 and 85\%. Similarly, ESA-responses were modulated during double conditions in the direction of levels observed for the attended stimulus presented alone, but to a lesser extent as indicated by the smaller AMI of $68 \%$. Contrary to these qualitatively similar dependencies of $\gamma$-PhC and ESA-responses on the attended stimulus, both measures behaved qualitatively different prior to behavioral errors. ESAresponses in periods preceding false alarms were very similar to the responses observed prior to correctly terminated trials. In contrast, high levels of ESA-LFP $\gamma$-synchronization were strongly reduced in error trials. A similar pattern was observed for the comparison of misses and correctly executed trials shown for the monkey with a sufficient number of misses (SF 2). Furthermore, the $\gamma$-PhC between the ESAs of two groups of neurons where at least one site responded poorly to the attended stimulus, or very similar for both stimuli, strongly reduced when a distractor was added. These observations suggest that attention configures the pattern of functional coupling between the networks neurons specifically for effective processing of the relevant stimulus. Furthermore, our findings point to a topdown mechanism, which works in parallel to mechanisms gating relevant information to downstream areas and configures the synchronization within local network.

The results of attention-dependent modulations of spiking activity are well in line with previous work. Our task paradigm evoked different spiking activity for the two stimuli based on differences in location instead of orientation or motion direction as in previous studies. Furthermore, the stimuli were attended in conditions with and without distractor. Nevertheless the observed ESA-responses showed an attention-dependent modulation which was very similar to previous results (Moran and Desimone, 1985; Treue and Maunsell, 1996; Luck et al., 1997; Reynolds et al., 1999; Lee and Maunsell, 2010; Ni et al., 2012). Furthermore, a dependence of local synchronization on different stimulus configurations has been observed for anesthetized cats (Espinosa and Gerstein, 1988; Engel et al., 1991; Zhou et al., 2008) and for monkey under passive viewing conditions (Kreiter and Singer, 1996b; Frien et al., 2000; Maldonado, 2000). The latter results support the notion that processing of specific stimuli is associated with a specific pattern of synchronization within a local neuronal network, which is thought to reflect the functional coupling within this network.

However, we were interested in whether attention invokes stimulus-specific network configurations depending on the momentary behavioral demand, even though the stimulus input does not change. These attention-dependent changes would allow for an extensive number of different network configurations within the framework of the given anatomical connections by selectively modulating the strength of functional coupling between the network's neurons (Aertsen et al., 1989; Kreiter and Singer, 1996a; Fries, 2005; Kreiter, 2006; Gregoriou et al., 2009; Battaglia et al., 2012; Palmigiano et al., 2017). If such a specific pattern of $\gamma$-synchronization within a local network is crucial for its ability to process a specific, behaviorally relevant stimulus, the pattern should change as attention switches between stimuli. Furthermore, it should be very similar to the pattern observed in the absence of the distractor stimulus. Our results confirm this prediction. When instead of a single stimulus two closely spaced stimuli provided input signals to a local neuronal network in $\mathrm{V} 4$, the $\gamma$-PhC depended on which stimulus was attended and was very similar to the values observed when this stimulus was presented alone. The AMI of $85 \%$ for the $\gamma$-PhC between ESA of two groups of neurons of the same local network illustrates the high precision by which selective attention adjusts the functional coupling strengths when the same stimulus is attended. The significantly weaker AMI for the ESA-responses support the notion that response strength and $\gamma-\mathrm{PhC}$ are not trivial consequences of each other, but reflect different aspects of neural processing (Bichot et al., 2005; Buffalo et al., 2011).

The hypothesis of attention-dependent dynamic network configuration by $\gamma$-band synchronization is further supported by 
pairs, which do not preserve their high $\gamma$-PhC for an attended stimulus when a distractor stimulus is added. Of those pairs, at least one site responds either very weakly to the attended stimulus or relatively strong to the distractor stimulus. These neurons are likely to receive a comparably high proportion of input signals representing the distractor, since even an attentiondependent input gating mechanism suppresses distractor related signals only to a limited extent. The reduced strength of functional coupling between those neurons and neurons of the dynamically defined network processing the attended stimulus should therefore help to avoid interference of distractor signals with processing of the attended stimulus. If in contrast only one stimulus is present, even weakly driven neurons can contribute to a network processing this stimulus since they carry no signals of distractors that could interfere. Well in line Vinck et al. (2013) gave evidence for a decoupling of those neurons from the processing network, which provided only poor information about an attended stimulus, even though no nearby distractor was present.

The relevance of $\gamma$-synchronization for the functional configuration of the local neuronal network in V4 is further supported by the characteristics of neuronal activity directly preceding behavioral errors. While the attention-dependent modulation of ESA-responses were almost unchanged in comparison to correctly executed trials, strong $\gamma$ synchronization between groups of neurons and the local population activity (LFP) in correctly executed trials vanished before an error. Several other studies also reported correlations between behavioral performance and oscillatory power or synchronization as well as firing-rates. In contrast to our study, they either used a task with only one stimulus in the RF to investigate error-dependent differences of synchronization (Tallon-Baudry et al., 2004), or compared conditions with attention directed into the RF versus away from the RF (Womelsdorf et al., 2006; Gregoriou et al., 2014). The latter two studies report that firing rates and synchronization show qualitatively similar differences when comparing either slow and fast reaction times (Womelsdorf et al., 2006) or error-dependent changes (Gregoriou et al., 2014). The level of synchronization and the firing rates were higher during fast trials as compared to slow trials and during correctly executed trials when compared to erroneously terminated trials. However, for our specific behavioral paradigm, recording constellation, and the attentional conditions compared here (attention directed always to a stimulus within the pRF of the recorded V4 population), we observed qualitatively different results for firing rates and $\gamma$-synchronization.

To put these results into perspective, we briefly recapitulate the selective gating of relevant information between and within visual areas. Previous work showed that V4 neurons synchronize selectively with afferent V1 neurons representing the attended stimulus while desynchronizing with those representing distractors (Bosman et al., 2012; Grothe et al., 2012). Furthermore, we showed that mainly signals carrying specific signatures related to the attended stimulus enter into the local processing network in V4.(Grothe et al., 2018). These findings, together with theoretical investigations, point toward an attention-dependent routing mechanism based on highly selective changes of functional coupling between V4 neurons and different subsets of their afferent inputs (Börgers and Kopell, 2008; Masuda, 2009; Tiesinga and Sejnowski, 2010; Battaglia et al., 2012; Hahn et al., 2014; Harnack et al., 2015). The almost unchanged level of firing rates during error and correctly performed trials in both task conditions, as shown in Figure 4A, indicate that attention was correctly directed to the target stimulus: If monkeys had attended the nearby distractor in the same pRF, the firing rates should have approached the strength associated with the distractor. A similar consideration holds if the animals would have allocated attention elsewhere. In this case, one would expect an intermediate firing rate, as observed for trials with attention directed away from the pRF $\left(\mathrm{D}^{0}\right.$-condition, Figure 2B). Our findings therefore indicate that those attention-dependent top-down mechanisms that selectively route the signals of the attended stimulus from upstream areas to the V4 neurons, were unlikely the source of the error.

The degradation of local $\gamma$-synchronization preceding errors rather indicates that successful processing of an attended stimulus also depends on a specific synchronization in the supragranular layers of V4. The recorded neurons in the upper layers of V4 show the expected level of spiking activity and are therefore likely to receive the correctly selected signals from the granular layer of V4. Thus, their local $\gamma$ synchronization is unlikely to depend solely on successful gating of the afferent stimulus related signals to V4. Rather, it is subject to attention-dependent top-down mechanisms, independent of a gating mechanism for the afferent bottomup input to V4. In line with our findings, Vinck and Bosman (2016) concluded in a recent review based on experimental data, that $\gamma$-oscillations in superficial and in granular layers can be generated fairly independent of each other. Thus, our observed strong degradation of $\gamma$-synchronization within the local network in superficial layers during error trials, might arise from a failure of attention-dependent top-down mechanisms to synchronize the neuronal network in layer $2 / 3$. At the same time, attentional mechanisms are still successful in selective gating of relevant information to layer 4 neurons. However, since neurons located in supragranular layers provide the corticocortical output projections, the disappearance of their strong $\gamma$ synchronization results in a reduced impact of their spikes on down-stream neurons (Niebur et al., 1993). Thus, the signal-tonoise ratio of the behaviorally relevant signal, and therefore its processing further downstream, is expected to be compromised. This may well give rise to erroneous responses (Taylor et al., 2005; Womelsdorf et al., 2006; Martin and von der Heydt, 2015) and might explain the different correlations between local $\gamma$ synchronization and firing-rates with behavioral outcome.

\section{CONCLUSION}

In summary, our results show clear similarities as well as specific differences between the attention-dependent modulation of $\gamma$-synchronization and spiking activity in local neuronal 
networks of area V4. These results are well in line with the expectations for an attention-dependent mechanism that structures functional coupling strengths and hence the functional configuration of a local network by modulating $\gamma$-band synchronization. We conclude that: (1) Attention adjusts the pattern of functional coupling strengths within a local neuronal network specifically for processing of an attended stimulus. (2) Attention dynamically decouples neurons from a network processing the attended stimulus when they would compromise processing with additional distractor related signals. (3) Degraded synchronization within the local network occurs just before behavioral errors in spite of almost intact attentiondependent firing-rates, indicating an error-location beyond afferent signal gating.

\section{AUTHOR CONTRIBUTIONS}

AK, SM, and IG designed the experiment. ED and $\mathrm{MH}$ performed the experiment and analysis. ED and AK wrote the manuscript. $\mathrm{SM}, \mathrm{MH}$, and IG discussed and reviewed the manuscript.

\section{REFERENCES}

Aertsen, A. M., Gerstein, G. L., Habib, M. K., and Palm, G. (1989). Dynamics of neuronal firing correlation: modulation of "effective connectivity". J. Neurophysiol. 61, 900-917. doi: 10.1152/jn.1989.61.5.900

Azouz, R., and Gray, C. M. (2003). Adaptive coincidence detection and dynamic gain control in visual cortical neurons in vivo. Neuron 37, 513-523. doi: 10.1016/S0896-6273(02)01186-8

Battaglia, D., Witt, A., Wolf, F., and Geisel, T. (2012). Dynamic effective connectivity of inter-areal brain circuits. PLoS Comput. Biol. 8:e1002438. doi: 10.1371/journal.pcbi.1002438

Bichot, N. P., Rossi, A. F., and Desimone, R. (2005). Parallel and serial neural mechanisms for visual search in macaque area V4. Science 308, 529-534. doi: 10.1126/science. 1109676

Börgers, C., and Kopell, N. J. (2008). Gamma osillations and stimulus selection. Neural Comput. 20, 383-414. doi: 10.1162/neco.2007.07-06-289

Bosman, C., Schoffelen, J. M., Brunet, N., Oostenveld, R., Bastos, A. M., Womelsdorf, T., et al. (2012). Attentional stimulus selection through selective synchronization between monkey visual areas. Neuron $75,875-888$. doi: 10.1016/j.neuron.2012.06.037

Brosch, M., Bauer, R., and Eckhorn, R. (1997). Stimulus-dependent modulations of correlated high-frequency oscillations in cat visual cortex. Cereb. Cortex. 7 , 70-76. doi: 10.1093/cercor/7.1.70

Buffalo, E. A., Fries, P., Landman, R., Buschman, T. J., and Desimone, R. (2011). Laminar differences in gamma and alpha coherence in the ventral stream. Proc. Natl. Acad. Sci. U.S.A. 108, 11262-11267. doi: 10.1073/pnas.1011284108

Carandini, M., and Heeger, D. J. (2012). Normalization as a canonical neural computation. Nat. Rev. Neurosci. 13, 51-62. doi: 10.1038/nrn3136

Carter, G., Knapp, C. H., and Nuttall, A. H. (1973). Estimation of the magnitudesquared coherence function via overlapped fast Fourier transform processing. IEEE Trans. Audio Electroacoust. 21, 337-344. doi: 10.1109/TAU.1973.11 62496

Cohen, M. R., and Maunsell, J. H. R. (2009). Attention improves performance primarily by reducing interneuronal correlations. Nat. Neurosci. 12, 1594-1600. doi: $10.1038 / \mathrm{nn} .2439$

Desimone, R. (1998). Visual attention mediated by biased competition in extrastriate visual cortex. Philos. Trans. R. Soc. Lond. B. Biol. Sci. 353, 1245-1255. doi: 10.1098/rstb.1998.0280

\section{FUNDING}

The Bernstein Group for Computational Neuroscience Bremen (Grant 01GQ0705) and the German Research Foundation (Grant KR 1844/4-1) have supported this work. Furthermore, ED was supported by the Fazit-Stiftung and IG was supported by the Leibniz Graduate School for Primate Neurobiology.

\section{ACKNOWLEDGMENTS}

We like to thank Aleksandra Nadolski for the support and valuable help with animal training and experiments, as well as Katrin Thoß and Ramazani Hakizimana for animal care. We also thank Dr. Detlef Wegener and Bastian Schledde for helpful comments on the manuscript.

\section{SUPPLEMENTARY MATERIAL}

The Supplementary Material for this article can be found online at: https://www.frontiersin.org/articles/10.3389/fncir. 2018.00071/full\#supplementary-material

Engel, A. K., Gerloff, C., Hilgetag, C. C., and Nolte, G. (2013). Intrinsic coupling modes: multiscale interactions in ongoing brain activity. Neuron 80, 867-886. doi: 10.1016/j.neuron.2013.09.038

Engel, A. K., Kreiter, A. K., König, P., and Singer, W. (1991). Synchronization of oscillatory neuronal responses between striate and extrastriate visual cortical areas of the cat. Proc. Natl. Acad. Sci. U.S.A. 88, 6048-6052. doi: $10.1073 /$ pnas.88.14.6048

Espinosa, I. E., and Gerstein, G. L. (1988). Cortical auditory neuron interactions during presentation of 3-tone sequences: effective connectivity. Brain Res. 450, 39-50. doi: 10.1016/0006-8993(88)91542-9

Frien, A., Eckhorn, R., Bauer, R., Woelbern, T., and Gabriel, A. (2000). Fast oscillations display sharper orientation tuning than slower components of the same recordings in striate cortex of the awake monkey. Eur. J. Neurosci. 12, 1453-1465. doi: 10.1046/j.1460-9568.2000.00025.x

Fries, P. (2005). A mechanism for cognitive dynamics: neuronal communication through neuronal coherence. Trends Cogn. Sci. 9, 474-480. doi: 10.1016/j.tics.2005.08.011

Gail, A., Brinksmeyer, H. J., and Eckhorn, R. (2000). Contour decouples gamma activity across texture representation in monkey striate cortex. Cereb. Cortex 10, 840-850. doi: 10.1093/cercor/10.9.840

Galashan, F. O., Saßen, H. C., Kreiter, A. K., and Wegener, D. (2013). Monkey area MT latencies to speed changes depend on attention and correlate with behavioral reaction times. Neuron 78, 740-750. doi: 10.1016/j.neuron.2013.03.014

Gilbert, C. D., and Sigman, M. (2007). Brain states: top-down influences in sensory processing. Neuron 54, 677-696. doi: 10.1016/j.neuron.2007.05.019

Gregoriou, G. G., Gotts, S. J., Zhou, H., and Desimone, R. (2009). High-frequency, long-range coupling between prefrontal and visual cortex during attention. Science 324, 1207-1210. doi: 10.1126/science.1171402

Gregoriou, G. G., Rossi, A. F., Ungerleider, L. G., and Desimone, R. (2014). Lesions of prefrontal cortex reduce attentional modulation of neuronal responses and synchrony in V4. Nat. Neurosci. 17, 1003-1011. doi: 10.1038/ nn. 3742

Grothe, I., Neitzel, S. D., Mandon, S., and Kreiter, A. K. (2012). Switching neuronal inputs by differential modulations of gamma-band phase-coherence. J. Neurosci. 32, 16172-16180. doi: 10.1523/JNEUROSCI.0890-12.2012

Grothe, I., Rotermund, D., Neitzel, S. D., Mandon, S., Ernst, U. A., Kreiter, A. K., et al. (2018). Attention selectively gates afferent signal transmission 
to area V4. J. Neurosci. 38, 3441-3452. doi: 10.1523/JNEUROSCI.222117.2018

Hahn, G., Bujan, A. F., Frégnac, Y., Aertsen, A., and Kumar, A. (2014). Communication through resonance in spiking neuronal networks. PLoS Comput. Biol. 10:e1003811. doi: 10.1371/journal.pcbi.1003811

Harnack, D., Ernst, U. A., and Pawelzik, K. R. (2015). A model for attentional information routing through coherence predicts biased competition and multistable perception. J. Neurophysiol. 114, 1593-1605. doi: $10.1152 /$ jn. 01038.2014

König, P., and Schillen, T. B. (1991). Stimulus-dependent assembly formation. Neural Comput. 3, 155-166.

Kreiter, A. K. (2006). How do we model attention-dependent signal routing? Neural Netw. 19, 1443-1444. doi: 10.1016/j.neunet.2006.09.005

Kreiter, A. K., and Singer, W. (1996a). On the role of neural synchrony in the primate visual cortex. Brain Theory Biol. Basis Comput. Princ. 1, 201-227. doi: 10.1016/B978-044482046-4/50053-5

Kreiter, A. K., and Singer, W. (1996b). Stimulus-dependent synchronization of neuronal responses in the visual cortex of the awake macaque monkey. J. Neurosci. 16, 2381-2396. doi: 10.1523/JNEUROSCI.16-07-023 81.1996

Lachaux, J. P., Rodriguez, E., Martinerie, J., and Varela, F. J. (1999). Measuring phase synchrony in brain signals. Hum. Brain Mapp. 8, 194-208. doi: 10.1002/ (SICI) 1097-0193(1999)8:4<194::AID-HBM4>3.0.CO;2-C

Lee, J., and Maunsell, J. H. R. (2010). Attentional modulation of MT neurons with single or multiple stimuli in their receptive fields. J. Neurosci. 30, 3058-3066. doi: 10.1523/JNEUROSCI.3766-09.2010

Legatt, A. D., Arezzo, J., and Vaughan, H. G. (1980). Averaged multiple unit activity as an estimate of phasic changes in local neuronal activity: effects of volume-conducted potentials. J. Neurosci. Methods 2, 203-217. doi: 10.1016/0165-0270(80)90061-8

Luck, S. J., Chelazzi, L., Hillyard, S. A., and Desimone, R. (1997). Neural mechanisms of spatial selective attention in areas V1, V2, and V4 of macaque visual cortex. J. Neurophysiol. 77, 24-42. doi: 10.1152/jn.1997.77.1.24

Maldonado, P. E. (2000). Dynamics of striate cortical activity in the alert macaque: II. Fast time scale synchronization. Cereb. Cortex 10, 1117-1131. doi: $10.1093 /$ cercor/10.11.1117

Martin, A. B., and von der Heydt, R. (2015). Spike synchrony reveals emergence of proto-objects in visual cortex. J. Neurosci. 35, 6860-6870. doi: 10.1523/JNEUROSCI.3590-14.2015

Masuda, N. (2009). Selective population rate coding: a possible computational role of gamma oscillations in selective attention. Neural Comput. 21, 3335-3362. doi: 10.1162/neco.2009.09-08-857

McAdams, C. J., and Maunsell, J. H. (1999). Effects of attention on orientationtuning functions of single neurons in macaque cortical area V4. J. Neurosci. 19, 431-441. doi: 10.1523/JNEUROSCI.19-01-00431.1999

Mirabella, G., Bertini, G., Samengo, I., Kilavik, B. E., Frilli, D., Della Libera, C., et al. (2007). Neurons in area V4 of the macaque translate attended visual features into behaviorally relevant categories. Neuron 54, 303-318. doi: 10.1016/j.neuron.2007.04.007

Mitchell, J. F., Sundberg, K. A., and Reynolds, J. H. (2007). Differential attentiondependent response modulation across cell classes in macaque visual area V4. Neuron 55, 131-141. doi: 10.1016/j.neuron.2007.06.018

Mitchell, J. F., Sundberg, K. A., and Reynolds, J. H. (2009). Spatial attention decorrelates intrinsic activity fluctuations in macaque area V4. Neuron 63, 879-888. doi: 10.1016/j.neuron.2009.09.013

Moran, J., and Desimone, R. (1985). Selective attention gates visual processing in the extrastriate cortex. Science 229, 782-784. doi: 10.1126/science.40 23713

Ni, A. M., Ray, S., and Maunsell, J. H. R. (2012). Tuned normalization explains the size of attention modulations. Neuron 73, 803-813. doi: 10.1016/j.neuron.2012.01.006

Niebur, E., Koch, C., and Rosin, C. (1993). An oscillation-based model for the neuronal basis of attention. Vision Res. 33, 2789-2802. doi: 10.1016/0042-6989(93)90236-P

Palmigiano, A., Geisel, T., Wolf, F., and Battaglia, D. (2017). Flexible information routing by transient synchrony. Nat. Neurosci. 20, 1014-1022. doi: $10.1038 / \mathrm{nn} .4569$
Park, H. J., and Friston, K. J. (2013). Structural and functional brain networks: from connections to cognition. Science 342:1238411. doi: 10.1126/science.12 38411

Perkel, D. H., Gerstein, G. L., and Moore, G. P. (1967). Neuronal spike trains and stochastic point processes: II. Simultaneous spike trains. Biophys. J. 7, 419-440.

Reynolds, J. H., Chelazzi, L., and Desimone, R. (1999). Competitive mechanisms subserve attention in macaque areas V2 and V4. J. Neurosci. 19, 1736-1753. doi: 10.1523/JNEUROSCI.19-05-01736.1999

Reynolds, J. H., Pasternak, T., and Desimone, R. (2000). Attention increases sensitivity of V4 neurons. Neuron 26, 703-714. doi: 10.1016/ S0896-6273(00)81206-4

Schledde, B., Galashan, F. O., Przybyla, M., Kreiter, A. K., and Wegener, D. (2017). Task-specific, dimension-based attentional shaping of motion processing in monkey area MT. J. Neurophysiol. 118, 1542-1555. doi: 10.1152/jn.001 83.2017

Schroeder, C. E., Tenke, C. E., Givre, S. J., Arezzo, J. C., and Vaughan, H. G. (1991). Striate cortical contribution to the surface-recorded pattern-reversal vep in the alert monkey. Vision Res. 31, 1143-1157. doi: 10.1016/0042-6989(91)90040-C

Segev, I., and Rall, W. (1998). Excitable dendrites and spines: earlier theoretical insights elucidate recent direct observations. Trends Neurosci. 21, 453-460. doi: 10.1016/S0166-2236(98)01327-7

Singer, W. (1993). Synchronization of cortical activity and its putative role in information processing and lerning. Annu. Rev. Physiol. 55, 349-374. doi: $10.1038 / \mathrm{nn} .3413 . \mathrm{A}$

Supér, H., and Roelfsema, P. R. (2005). Chronic multiunit recordings in behaving animals: advantages and limitations. Prog. Brain Res. 147, 263-282. doi: 10.1016/S0079-6123(04)47020-4

Tallon-Baudry, C., Bertrand, O., Delpuech, C., and Pernier, J. (1997). Oscillatory $\gamma$ band $(30-70 \mathrm{~Hz})$ activity induced by a visual search task in humans. J. Neurosci. $17,722-734$.

Tallon-Baudry, C., Mandon, S., Freiwald, W. A., and Kreiter, A. K. (2004). Oscillatory synchrony in the monkey temporal lobe correlates with performance in a visual short-term memory task. Cereb. Cortex 14, 713-720. doi: 10.1093/cercor/bhh031

Taylor, K., Mandon, S., Freiwald, W. A., and Kreiter, A. K. (2005). Coherent oscillatory activity in monkey area $\mathrm{v} 4$ predicts successful allocation of attention. Cereb. Cortex 15, 1424-1437. doi: 10.1093/cercor/bhi023

Tiesinga, P. H., and Sejnowski, T. J. (2010). Mechanisms for phase shifting in cortical networks and their role in communication through coherence. Front. Hum. Neurosci. 4:196. doi: 10.3389/fnhum.2010.00196

Torrence, C., and Compo, G. P. (1998). A practical guide to wavelet analysis. Bull. Am. Meteorol. Soc. 79, 61-78. doi: 10.1175/1520-0477(1998)079<0061:APGTWA>2.0.CO;2

Treue, S., and Martínez Trujillo, J. C. (1999). Feature-based attention influences motion processing gain in macaque visual cortex. Nature 399, 575-579. doi: $10.1038 / 21176$

Treue, S., and Maunsell, J. H. (1996). Attentional modulation of visual motion processing in cortical areas MT and MST. Nature 382, 539-541. doi: $10.1038 / 382539 \mathrm{a} 0$

Usrey, W. M., Alonso, J. M., and Reid, R. C. (2000). Synaptic interactions between thalamic inputs to simple cells in cat visual cortex. J. Neurosci. 20, 5461-5467. doi: 10.1523/JNEUROSCI.20-14-05461.2000

Vinck, M., and Bosman, C. A. (2016). More gamma more predictions: gammasynchronization as a key mechanism for efficient integration of classical receptive field inputs with surround predictions. Front. Syst. Neurosci. 10:35. doi: 10.3389/fnsys.2016.00035

Vinck, M., Womelsdorf, T., Buffalo, E., Desimone, R., and Fries, P. (2013). Attentional modulation of cell-class-specific gamma-band synchronization in awake monkey area V4. Neuron 80, 1077-1089. doi: 10.1016/j.neuron.2013.08.019

Wegener, D., Freiwald, W. A., and Kreiter, A. K. (2004). The influence of sustained selective attention on stimulus selectivity in macaque visual area MT. J. Neurosci. 24, 6106-6114. doi: 10.1523/JNEUROSCI.1459-04.2004

Wildie, M. (2012). Establishing communication between neuronal populations through competitive entrainment. Front. Comput. Neurosci. 5, 1-16. doi: $10.3389 /$ fncom.2011.00062 
Womelsdorf, T., Fries, P., Mitra, P. P., and Desimone, R. (2006). Gamma-band synchronization in visual cortex predicts speed of change detection. Nature 439, 733-736. doi: 10.1038/nature04258

Womelsdorf, T., Schoffelen, J.-M., Oostenveld, R., Singer, W., Desimone, R., Engel, A. K., et al. (2007). Modulation of neuronal interactions through neuronal synchronization. Science 316, 1609-1612. doi: 10.1126/science. 1139597

Zhou, Z., Bernard, M. R., and Bonds, A. B. (2008). Deconstruction of spatial integrity in visual stimulus detected by modulation of synchronized activity in cat visual cortex. J. Neurosci. 28, 3759-3768. doi: 10.1523/JNEUROSCI.448107.2008
Conflict of Interest Statement: The authors declare that the research was conducted in the absence of any commercial or financial relationships that could be construed as a potential conflict of interest.

Copyright $(2018$ Drebitz, Haag, Grothe, Mandon and Kreiter. This is an open-access article distributed under the terms of the Creative Commons Attribution License (CC $B Y)$. The use, distribution or reproduction in other forums is permitted, provided the original author(s) and the copyright owner(s) are credited and that the original publication in this journal is cited, in accordance with accepted academic practice. No use, distribution or reproduction is permitted which does not comply with these terms. 